\title{
6. THE TOTAL BRIGHTNESS OF GALACTIC CLUSTERS
}

\section{W. Buscombe \\ Mount Stromlo Observatory}

For many generations astronomers have been fascinated by open clusters of stars, of which many are found close to the central plane of the Milky Way. Only in comparatively recent years, however, has it become possible to derive reasonably consistent distances for these stellar systems. The difficulty was that until techniques of photoelectric photometry in at least three colours were developed, there were no reliable estimates of the amount of interstellar absorption and reddening.

All extensive catalogues of clusters which were compiled prior to 1960 give rather unsatisfactory estimates of the distances and linear diameters of clusters. Most of these data were based on the assumption that all clusters have either the same linear diameter or the same luminosity function. From fitting the main sequence (and possibly also the giant branch) of the diagram of intrinsic colour and correct apparent magnitude for the stars in a cluster to the standard graph of colour and absolute magnitude for nearby stars with accurately determined parallaxes, Johnson and others have derived photometric distance moduli for more than 150 clusters. These are summarized in Mount Stromlo Mimeogram No. 6, where general references are also quoted. Becker (this volume, paper 3 ) indicates how this procedure can be made more exact with two colour-indices.

After the frequency distributions of stars with different intrinsic brightness were found to be different in typical clusters, Sandage and others showed that the clusters containing supergiants and hot blue stars are younger than those dominated by stars of the giant branch.

For some clusters containing stars brighter than the eighth magnitude, luminosity classifications of a few stars have been made from slit spectrograms. These data aid the analyst of photometric data to assign intrinsic colours, especially for giant and supergiant stars, but as yet the calibration of absolute magnitudes for supergiants is not completely consistent. Since it is difficult in practice to estimate the degree of evolution of hot stars above the main sequence, spectroscopic distance moduli for the brightest individual stars may give an underestimate of the distance for the cluster.

This paper will examine how one can estimate the intrinsic brightness of a cluster, mainly with photometric observations, and thus obtain its distance. The estimates of integrated apparent brightness, expressed on the scale of photographic magnitude by Lundmark and Collinder about 1930, were converted to absolute photographic magnitude for all clusters whose distance and interstellar reddening have been determined from photoelectric measures in three colours.

It would have been preferable to use integrated photoelectric magnitudes, but these are available for very few clusters, mainly in extragalactic systems such as the Magellanic Clouds and Messier 31. In the later section the significance of integrated colours, which have been measured for a few clusters with wide-field photometers, will be discussed. 
The main characteristics of galactic clusters were examined by grouping their integrated absolute magnitudes in steps of $\Delta M=1 \cdot 5$, i.e. intervals of a factor 4 in total brightness from one group to the next. Except for the very brightest clusters, of which there are only a few representatives, the average absolute magnitude $\left(M_{B}\right)$ for the brightest star in each cluster is very well correlated with the integrated

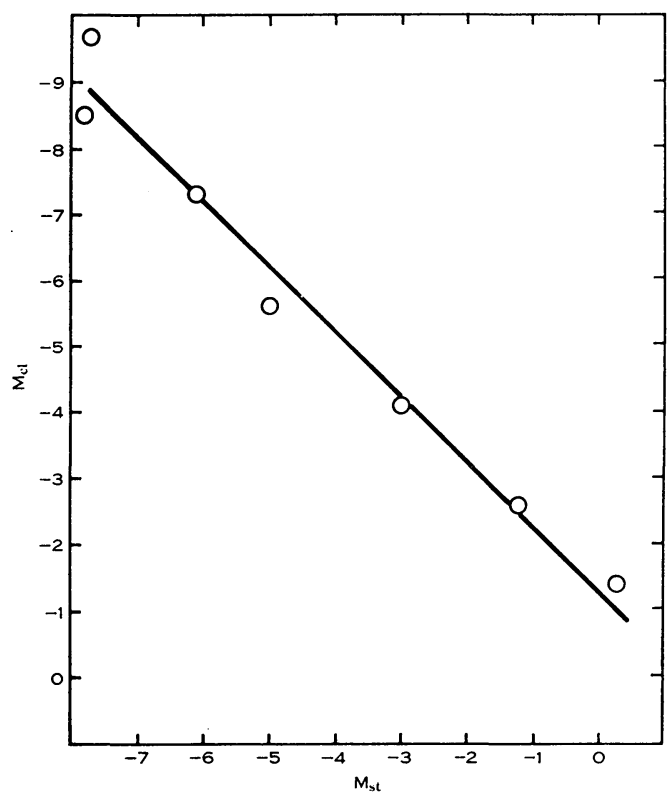

Fig. 1.-Mean relation between total brightness of cluster (ordinate) and absolute magnitude of brightest star.

TABLE 1

AVERAGED DATA FOR GROUPS OF CLUSTERS

\begin{tabular}{|c|c|c|c|c|c|c|c|}
\hline $\bar{M}_{c l}$ & \multicolumn{2}{|c|}{$\bar{r} \pm$ p.e. $(n) \quad(\mathrm{kpc})$} & \multicolumn{2}{|c|}{$\bar{M}_{s t} \pm$ p.e. $(n)$} & \multicolumn{2}{|c|}{$\bar{d} \pm$ p.e. $(\mathrm{pc})$} & $\log \bar{d}$ \\
\hline$-9 \cdot 7$ & $2 \cdot 2$ & $0 \cdot 07 \quad(4)$ & $-7 \cdot 7$ & 0.07 & 20 & 3 & $1 \cdot 20$ \\
\hline$-8 \cdot 5$ & $2 \cdot 1$ & $0 \cdot 10(10)$ & $-7 \cdot 8$ & $0 \cdot 10$ & 10 & $1 \cdot 4$ & 0.99 \\
\hline$-7 \cdot 3$ & $2 \cdot 04$ & $0 \cdot 12(21)$ & $-6 \cdot 1$ & $0.04(18)$ & 9 & 0.9 & 0.95 \\
\hline$-5 \cdot 6$ & $1 \cdot 62$ & $0 \cdot 10(34)$ & $-5 \cdot 0$ & $0.08(26)$ & $6 \cdot 2$ & 0.4 & $0 \cdot 79$ \\
\hline$-4 \cdot 1$ & $1 \cdot 20$ & $0.04(48)$ & $-3 \cdot 0$ & $0 \cdot 13(24)$ & $5 \cdot 4$ & $0 \cdot 14$ & 0.73 \\
\hline$-2 \cdot 6$ & 0.89 & $0.08(27)$ & $-1 \cdot 2$ & $0 \cdot 19(15)$ & $3 \cdot 8$ & 0.22 & 0.58 \\
\hline$-1 \cdot 4$ & 0.8 & $0 \cdot 10(13)$ & +0.3 & $0 \cdot 17 \quad(6)$ & $3 \cdot 0$ & $0 \cdot 3$ & 0.48 \\
\hline
\end{tabular}

photographic absolute magnitude. The linear coefficient is $0 \cdot 755 \pm 0 \cdot 042$ (s.e.). In most clusters a single star, or else a small group of stars equally bright, contributes the majority of the light. Figure 1 and Table 1 indicate the mean relation.

Another significant correlation is found - that the average linear diameter declines monotonically with the total brightness for each group of clusters. The 
equation of the linear relation in Figure 2 is $\log d=0 \cdot 38-0 \cdot 080 M$. However, a definite effect of selection is seen through the course of the average distances. For the most luminous clusters, the observational data may be nearly complete

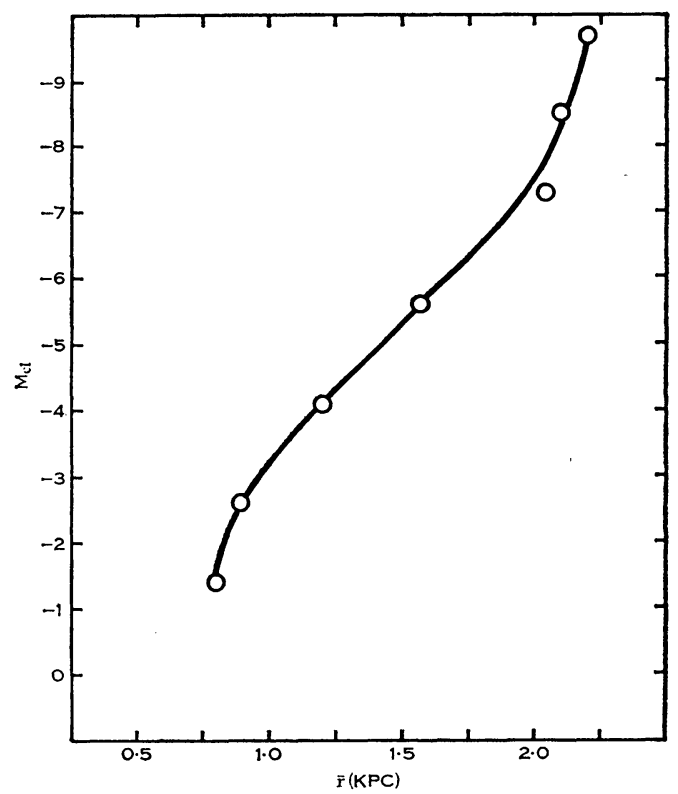

Fig. 2.-Observed relation between brightness of cluster and average distance.

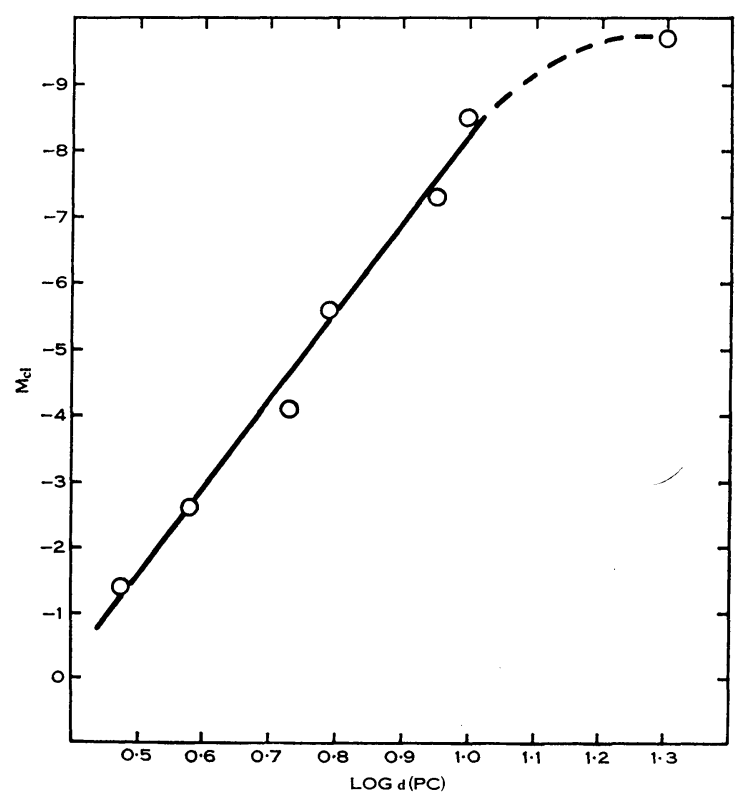

Fig. 3.-Mean linear diameters of clusters (abscissa), grouped according to total brightness. 
within a sphere of radius $3 \mathrm{kpc}$ around the Sun, but less luminous clusters have only been discovered and studied within about half that distance. (See Fig. 3.) No adjustment for background light has been attempted in this discussion.

It may be of some interest to note that the majority of very luminous clusters associated with emission nebulosity is found in the southern sky. On the other hand, information on the least luminous classes of cluster is as yet confined entirely to northern systems. Southern astronomers have recently made some headway with accurate photometric and spectroscopic studies of the brightest clusters, but have not such great reserves of instrumental facilities and manpower as have been built up in northern countries over many generations.

TABLE 2

BRIGHTEST STARS IN GALACTIC CLUSTERS

\begin{tabular}{|c|c|c|}
\hline $\bar{M}_{c l}$ & $\bar{M}_{s t}$ & Spectral Types of Brightest Stars \\
\hline$-9 \cdot 7$ & $-7 \cdot 7$ & O8: (IC 2944), B1 Ia-0 (NGC 6231), B2 Ia ( $\chi$ Per), Al Ia (h Per) \\
\hline$-8 \cdot 4$ & $-7 \cdot 7$ & O5f (2), O8f, B3 Ia, B6 Iab; A2 Ia, F0 Ia, G1 Ib \\
\hline$-7 \cdot 3$ & $-6 \cdot 1$ & $\begin{array}{l}\text { O5, O6 (3), O7 (2), O9 III, 09.5 Ia (2); B0 Ia (2), B0 Ib (3), B1 Ib, B2 Ia, } \\
\text { B3 Ia, B3 Iab, B3 Ib, B5 Ib }\end{array}$ \\
\hline$-5 \cdot 6$ & $-5 \cdot 2$ & $\begin{array}{l}\text { O6 (2), O7 (2), O8, O9 Ib, O9 III; B0 Ia, B0 II, B0 III, B0 IV, B0 V, B1 Ia, } \\
\text { B2 III, B9 Ib; F0 Ia, F5 Ia; K5 Ib; M1 Ib (II Sco) }\end{array}$ \\
\hline$-4 \cdot 1$ & $-3 \cdot 1$ & B0 V (2), B1 IV, B2 IV, B3 III, B3 V (2), B7 III (Pleiades), B8 III; G8 III \\
\hline$-2 \cdot 8$ & $-1 \cdot 9$ & B2 IV, B3 III, B5 III, B8 III; A7 III (Hyades); F0 III; K0 III (Praesepe) \\
\hline$-1 \cdot 4$ & $+0 \cdot 3$ & B8 III, B9 III; F0 III (Coma); G8 III; K1 III \\
\hline
\end{tabular}

A few further points emerge from consideration of the MK luminosity classes of the brightest individual stars in each of 73 clusters, summarized in Table 2. In each cluster having the integrated absolute magnitude brighter than -8 , the type of the brightest star is either Of or an early-type supergiant of class Ia. This survey confirms other evidence that the absolute visual magnitudes of Of stars are of the order -7 .

For clusters in the next groups, with $-8<M_{p g}<-5$, the characteristic types for the brightest individual stars are $\mathrm{Ib}$ supergiants and absorption-line $\mathrm{O}$ stars. For clusters with $-5<M_{p g}<-2$, early B-type stars on the main sequence and late-type supergiants appear among the brightest stars. Only clusters in the intrinsically faintest group considered are dominated by stars of the giant branch.

The available evidence suggests that the youngest clusters, dominated by a single early Of-type star, are the most compact, with diameters of only a parsec or less, embedded in more extensive nebulosity. In a later stage of evolution there may be one or several B-type supergiants in a cluster for which 15 or $20 \mathrm{pc}$ is a 
typical diameter. Emission nebulae are not reported around clusters whose integrated absolute magnitude is fainter than -5 . (See Table 3.) Incidentally, the brightest globular clusters, with absolute magnitudes around -9 , have diameters of $30 \mathrm{pc}$. Unfortunately, the individual diameters, especially of open clusters, are comparatively uncertain, and could be improved only by very detailed studies of star counts interpreted from measures of the fluctuating effect of interstellar absorption.

TABLE 3

DIAMETERS OF CLUSTERS AND NEBULOSITY

\begin{tabular}{|c|c|c|c|c|}
\hline$M_{c l}$ & NGC & $\begin{array}{c}d_{c l} \\
(\mathrm{pc})\end{array}$ & $\begin{array}{l}d_{n e b} \\
(\mathrm{pc})\end{array}$ & Constellation \\
\hline$-9 \cdot 8$ & 6231 & 10 & 150 & Sco \\
\hline$-9 \cdot 6:$ & IC 2944 & 30 & 50 & Car \\
\hline$-8 \cdot 9$ & 3572 & 20 & 20 & Car \\
\hline$-8 \cdot 8$ & 6611 & 6 & 30 & Ser \\
\hline$-8 \cdot 3$ & Cr 173 & 7 & 120 & Vel \\
\hline$-8 \cdot 2:$ & Cr 228 & 10 & 100 & Car \\
\hline$-8 \cdot 1$ & IC 1805 & 12 & 90 & Cas \\
\hline$-8 \cdot 0$ & 2244 & 12 & 50 & Mon \\
\hline$-7 \cdot 5$ & $1893 / \mathrm{IC} 410$ & 18 & 25 & Aur \\
\hline$-7 \cdot 5$ & 2362 & 3 & 220 & $\mathrm{CMa}$ \\
\hline$-7 \cdot 3$ & 6193 & 8 & 50 & Ara \\
\hline$-7 \cdot 1$ & IC 1848 & 14 & 30 & Cas \\
\hline$-7 \cdot 1$ & 2579 & 4 & 150 & Pup \\
\hline$-7 \cdot 1$ & 3293 & 6 & 30 & $\mathrm{Car}$ \\
\hline$-7 \cdot 0$ & 6514 & 14 & 14 & Sgr \\
\hline$-7 \cdot 0$ & $6523 / 6530$ & 21 & 40 & Sgr \\
\hline$-6 \cdot 4$ & 7380 & 5 & 10 & Cep \\
\hline$-6 \cdot 3$ & 6383 & 2 & 40 & Sgr \\
\hline$-6 \cdot 1$ & Cr 302 & 35 & 30 & Sco \\
\hline$-5 \cdot 7$ & Cr 69 & 8 & 8 & Ori \\
\hline$-5 \cdot 7$ & 2264 & 7 & 7 & Mon \\
\hline$-5 \cdot 3$ & 2175 & 8 & 15 & Gem \\
\hline
\end{tabular}

In an attempt to find a basis for interpreting the integrated colours for associations of stars in the Magellanic Clouds, I have removed the colour excess, which had been determined from photoelectric colour diagrams for galactic open clusters, from the integrated $(P-V)$ and $(V-I)$ colours measured through a wide-field photometer by Kron and his collaborators (see Table 4). Among the 28 clusters for which these data have been published, the colours for more than half are characteristic of late B- and early A-type stars on the main sequence. (Extension of this work to southern systems would be highly desirable.) However, certain clusters, e.g. IC 1805 and NGC 1502 , are conspicuously blue. Their colour-magnitude arrays show a section of the main sequence for $\mathrm{O}, \mathrm{B}$, and $\mathrm{A}$ stars extending through an interval of 7 or 8 magnitudes. In other words, the diagrams are characteristic of very young clusters, and correspond generally to Trumpler's class 3 for their extensive range of stellar magnitudes. On the other hand, examples of red galactic clusters are NGC 2682 and $7789-$ 
intrinsically faint, dominated by red giants which contribute most of the light. To these clusters Trumpler has assigned class 1, since so many of their stars have similar brightness. Presumably these are quite old stellar groups, whose more massive members are already burnt out.

TABLE 4

INTRINSIC INTEGRATED COLOURS OF CLUSTERS

\begin{tabular}{|c|c|c|c|}
\hline NGC & $M_{B}$ & $(B-V)_{\mathrm{o}}$ & $(V-I)_{\mathrm{o}}$ \\
\hline 869 & $-9 \cdot 8$ & $-0 \cdot 18$ & -0.43 \\
\hline 884 & $-9 \cdot 7$ & +0.06 & +0.49 \\
\hline 663 & $-8 \cdot 7$ & $-0 \cdot 15:$ & $+0 \cdot 20$ \\
\hline 457 & $-8 \cdot 6$ & +0.08 & +0.01 \\
\hline 7790 & $-7 \cdot 4$ & $+0 \cdot 04$ & +0.05 \\
\hline 1502 & $-7 \cdot 2$ & $-0 \cdot 31$ & -0.50 \\
\hline IC 1805 & $-7:$ & $-0 \cdot 31$ & $-0 \cdot 44$ \\
\hline 581 & $-6 \cdot 7$ & +0.02 & $+0 \cdot 40$ \\
\hline 7380 & $-6 \cdot 4$ & -0.22 & $-0 \cdot 32$ \\
\hline 637 & $-5 \cdot 9$ & $-0 \cdot 06$ & $-0 \cdot 22$ \\
\hline 2099 & $-5 \cdot 5$ & $+0 \cdot 22$ & $+0 \cdot 12$ \\
\hline 1960 & $-5 \cdot 0$ & $-0 \cdot 16$ & $-0 \cdot 28$ \\
\hline 7789 & $-5 \cdot 0$ & +0.65 & +0.68 \\
\hline 1027 & $-4 \cdot 7$ & +0.09 & +0.02 \\
\hline 1912 & $-4 \cdot 7$ & +0.07 & +0.09 \\
\hline 559 & $-4 \cdot 3$ & $-0 \cdot 14$ & $+0 \cdot 19$ \\
\hline 1528 & $-4 \cdot 3$ & +0.05 & +0.09 \\
\hline IC 4725 & $-4 \cdot 2$ & $+0 \cdot 21$ & $+0 \cdot 35$ \\
\hline 6940 & $-3 \cdot 8$ & +0.31 & +0.86 \\
\hline 7243 & $-3 \cdot 8$ & $-0 \cdot 15$ & $-0 \cdot 28$ \\
\hline 7209 & $-3 \cdot 4$ & $+0 \cdot 30$ & +0.59 \\
\hline 6866 & $-3 \cdot 1$ & $+0 \cdot 26$ & $+0 \cdot 20$ \\
\hline $\operatorname{Tr} 2$ & $-3 \cdot 1$ & +0.06 & +0.40 \\
\hline 6633 & $-2 \cdot 7$ & $+0 \cdot 11$ & $-0 \cdot 20$ \\
\hline 2682 & $-2 \cdot 4$ & +0.69 & +0.63 \\
\hline 6811 & $-2 \cdot 4$ & +0.48 & +0.60 \\
\hline 7092 & $-2 \cdot 0$ & -0.03 & $-0 \cdot 24$ \\
\hline 1662 & $-1 \cdot 4$ & $+0 \cdot 15$ & $+0 \cdot 18$ \\
\hline
\end{tabular}

The integrated intrinsic colours of several open clusters in the northern Milky Way, computed by Arp for paper 50 in this Symposium, agree well with Kron's observations. However, the agreement is not yet so satisfactory in published data relating to the Large Magellanic Cloud.

It is a pleasure to thank Mr. Charles Dickens for his assistance in preparing this paper and Mount Stromlo Mimeogram No. 6. Professor G. E. Kron has graciously permitted the use of some unpublished magnitudes for clusters. 